\title{
ADESÃO AO PADRÃO ALIMENTAR MEDITERRÂNICO EM CRIANÇAS E ADOLESCENTES EM CONTEXTO DE CONSULTA DE VIGILÂNCIA DE SAÚDE
}

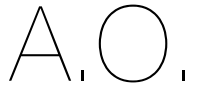

ARTIGO ORIGINAL

${ }^{1}$ Escola Superior

de Biotecnologia da

Universidade Católica

Portuguesa,

Rua Diogo de Botelho,

n. ${ }^{\circ} 1327$,

4169-005 Porto, Portugal

2 Centro da Criança e do Adolescente do Hospital CUF Porto,

Estrada da Circunvalação, n. ${ }^{\circ} 14341$,

4100-180 Porto, Portugal

${ }^{3}$ Centro de Biotecnologia e Química Fina da Escola Superior de Biotecnologia da Universidade Católica Portuguesa,

Rua Diogo de Botelho, n. ${ }^{1} 1327$,

4169-005 Porto, Portugal

${ }^{4}$ Ordem dos

Nutricionistas,

Rua do Pinheiro Manso,

n. ${ }^{\circ} 174$,

4100-409 Porto, Portugal

${ }^{5}$ Centro de Investigação em Tecnologias e Serviços de Saúde,

Rua Dr. Plácido da Costa, 4200-450 Porto, Portugal

${ }^{6}$ Faculdade de Medicina da Universidade do Porto, Rua Dr. Plácido da Costa, 4200-450 Porto, Portugal

*Endereço para correspondência:

Francisca Quaresma

Avenida da Praia, n. ${ }^{\circ} 403$ - Z4, 3885-404 Esmoriz, Portugal franciscarquaresma@gmail.com

Histórico do artigo:

Recebido a 19 de maio de 2020 Aceite a 30 de setembro de 2020

\begin{abstract}
ADHERENCE TO THE MEDITERRANEAN EATING PATTERN IN CHILDREN AND ADOLESCENTS IN THE CONTEXT OF HEALTH SURVEILLANCE CONSULTATION
\end{abstract}

Francisca Quaresma1"; Inês Tomada ${ }^{1-3}$; Rui da Silva4; Emídio Carreiro²; Carla Rêgo ${ }^{1,2,5,6}$

\section{RESUMO}

INTRODUÇÃo: O Padrão Alimentar Mediterrânico é um modelo alimentar de elevada qualidade e adequação nutricional, que se associa a melhor qualidade de vida. Portugal, embora seja um país com características mediterrânicas, está progressivamente a distanciar-se deste padrão, sobretudo em idades mais jovens, onde a adoção de hábitos alimentares saudáveis é essencial. OBJETIVOS: Avaliar a adesão ao Padrão Alimentar Mediterrânico em crianças e adolescentes, e a sua associação com o seu estado nutricional.

METODOLOGIA: Estudo transversal, descritivo e analítico. Foi aplicado o Mediterranean Diet Quality Index in Children and Adolescents (índice KIDMED), a uma amostra de conveniência de crianças e adolescentes, entre os 2 e os 18 anos, seguidos em consulta de vigilância de saúde num hospital privado do norte de Portugal. Para a caracterização do estado nutricional foi calculado o Índice de Massa Corporal e utilizados os critérios da Organização Mundial da Saúde. Foram critérios de exclusão uma idade inferior a 2 anos, qualquer tipo de vegetarianismo e a presença de doenças do comportamento alimentar.

RESULTADOS: Dos 153 inquiridos, com uma idade média de 9,9 4 4,3 anos e uma predominância do sexo feminino (56,2\%), 21,6\% apresentava excesso de peso, 18,9\% obesidade e 9,2\% baixo peso/desnutrição. Observou-se alta adesão ao Padrão Alimentar Mediterrânico (81,0\%), no entanto, esta era tanto menor quanto maior a idade $(r=0,279 ; p<0,001)$. Não se verificou relação entre a adesão a um padrão mediterrânico e o sexo ou o estado nutricional, nem com o momento de recolha dos dados (primeira consulta versus subsequente). Contudo, nas crianças e adolescentes com excesso de peso/obesidade observa-se um aumento da adesão nas consultas subsequentes.

CONCLUSÕES: A população estudada revela uma elevada adesão ao Padrão Alimentar Mediterrânico, registando-se, no entanto, uma diminuição com a idade. Crianças e adolescentes com excesso de peso/obesidade são mais recetivos a adotar este padrão alimentar.

\section{PALAVRAS-CHAVE}

KIDMED, Padrão Alimentar Mediterrânico, Pediatria

ABSTRACT

INTRODUCTION: The Mediterranean Eating Pattern is a food model of high quality and nutritional adequacy, which is associated with a better quality of life. Portugal, despite being a country with Mediterranean characteristics, is gradually moving away from this pattern. This occurs especially at younger ages, when the adoption of healthy eating habits is essential.

OBJECTIVES: To assess the adherence to the Mediterranean Eating Pattern in children and adolescents and its association with their nutritional status.

METHODOLOGY: Cross-sectional observational study. Mediterranean Eating Pattern was assessed using Mediterranean Diet Quality Index in Children and Adolescents (KIDMED index). Participants included children and adolescents aged 2-18 years, followed in health surveillance consultation at a private hospital in the north of Portugal. For the characterization of the nutritional status, the Body Mass Index was calculated, and the criteria of the World Health Organization were used. Exclusion criteria were defined as being less than 2 years old, any type of vegetarianism and the presence of diseases of eating behavior.

RESULTS: Of the 153 respondents, with an average age of 9,9 $9 \pm 4,3$ years and a predominance of girls (56,2\%), 21,6\% were overweight, $18,9 \%$ were obese and 9,2\% underweight/malnutrition. There was a high adherence to the Mediterranean Eating Pattern (81.0\%), although it decreased with age $(r=0.279 ; p<0.001)$. There was no relationship between adherence to a mediterranean pattern and gender or nutritional status, nor with the moment of data collection (first versus subsequent appointment). However, in overweight/ obese children and adolescents there is an increase in adherence in subsequent appointments.

CONCLUSIONS: The studied population shows a high adherence to the Mediterranean Eating Pattern, however, decreasing with age. Overweight/obese children and adolescents are more receptive to adopt this eating pattern.

KEYWORDS

KIDMED, Mediterranean Eating Pattern, Pediatric 


\section{INTRODUÇÃO}

O Padrão Alimentar Mediterrânico (PAM) caracteriza-se por um elevado consumo de alimentos de origem vegetal, tais como hortofrutícolas, leguminosas, frutos oleaginosos, cereais e azeite (utilizado como principal fonte de gordura da alimentação), e um consumo moderado de produtos de origem animal, nomeadamente pescado, carnes brancas, ovos e laticínios (1, 2). O baixo consumo de carnes vermelhas e de alimentos processados, a substituição do sal por ervas aromáticas, o consumo de vinho tinto às refeições principais (exceto por crianças, adolescentes e grávidas) e de água ao longo do dia, bem como preferência por produtos frescos, tradicionais, locais e sazonais são também pilares deste modelo alimentar (1, 2).

Atendendo às características climáticas, económicas, sociais, culturais e gastronómicas, Portugal é considerado um país com traços mediterrânicos (3). Porém, à semelhança de outros países, a população portuguesa está progressivamente a distanciar-se do PAM, particularmente em idades mais jovens (4-6).

A obesidade é atualmente um grave problema de saúde pública a nível mundial. Portugal não é exceção, sendo importante salientar a prevalência de excesso de peso e obesidade na sua população pediátrica. Segundo os dados do estudo COSI Portugal-2019, $29,6 \%$ das crianças portuguesas entre os 6 e os 8 anos apresentam excesso de peso, das quais 12,0\% são obesas (7). Já o Inquérito Alimentar Nacional e de Atividade Física 2015-1016 (IAN-AF) revelou uma prevalência de excesso de peso e obesidade de 17,3\% e 7,7\%, respetivamente, em crianças com idade inferior a 10 anos, e de 23,6\% e 8,7\% em adolescentes (dos 10 aos 17 anos) (8). A baixa qualidade da alimentação, especialmente a elevada ingestão de alimentos ricos em açúcar, gordura de origem animal e sal, e o inadequado aporte em vitaminas e minerais, provenientes de hortofrutícolas, aliado a um estilo de vida sedentário, são determinantes quer para as taxas de obesidade observadas, quer para o subsequente desenvolvimento de comorbilidades a curto e a médio prazos (9).

O PAM é um modelo alimentar de elevada qualidade e adequação nutricional (10). Possivelmente, em conjunto com um estilo de vida fisicamente ativo, é um dos padrões alimentares mais saudáveis, dada a sua associação a melhor qualidade de vida e a menores taxas de morbimortalidade por doenças crónicas não-transmissíveis, tais como cancro, diabetes mellitus tipo 2, obesidade e doença cardiovascular (11-14). A infância constitui uma oportunidade única para a aquisição de hábitos alimentares saudáveis, que se pretende que sejam mantidos ao longo da vida (15). O presente estudo teve como objetivo avaliar a adesão ao PAM, em crianças e adolescentes seguidos em consulta de Pediatria e de Nutrição Pediátrica num hospital privado do norte de Portugal, e a sua associação com o seu estado nutricional.

\section{METODOLOGIA}

Estudo transversal, descritivo e analítico, aplicado a uma amostra selecionada por conveniência de crianças e adolescentes, com idades compreendidas entre os 2 e os 18 anos, seguidos em consulta de vigilância de saúde ou de orientação nutricional num hospital privado do norte de Portugal. Foram considerados critérios de exclusão uma idade inferior a 2 anos, qualquer tipo de vegetarianismo e o diagnóstico de doenças do comportamento alimentar.

O protocolo de avaliação incluía a data de nascimento, o sexo, o tipo de consulta (Pediatria ou Nutrição Pediátrica), o momento de recolha da informação (primeira consulta ou consulta subsequente) e a medição do peso e da estatura. Procedeu-se ao cálculo do Índice de Massa Corporal (IMC) sendo utilizados os critérios da Organização Mundial da Saúde para a caracterização do estado de nutrição (16).
A adesão ao PAM foi avaliada através da aplicação do índice KIDMED, um questionário de fácil e rápida aplicação (11). O KIDMED é constituído por 16 questões, cada uma cotada positiva (+1) ou negativamente (-1), de acordo com a relação favorável ou não a este padrão alimentar, respetivamente. A cotação final deste questionário varia entre (-4) e (+12), sendo que uma pontuação $\leq 3$ revela fraca adesão, uma pontuação entre 4 e 7 reflete uma adesão intermédia e uma pontuação $\geq 8$ indica uma alta adesão ao PAM (17). O questionário foi aplicado de forma direta aos participantes ou, nas crianças mais novas, aos pais/cuidadores. Foi obtida a autorização do Conselho de Ética do Hospital CUF Porto para a aplicação do índice KIDMED - Mediterranean Diet Quality Index in Children and Adolescents - nas consultas de Pediatria e de Nutrição Pediátrica. Os pais/cuidadores foram informados dos objetivos, tendo dado a autorização para a recolha e o tratamento de dados.

Os dados foram analisados através do software Statistical Package for the Social Sciences (SPSS ${ }^{\circledR}$ ) versão 25. A análise baseou-se no cálculo das frequências relativas e absolutas e dos valores mínimos e máximos. Recorreu-se ao teste de independência do qui-quadrado para averiguar a relação de independência entre duas variáveis qualitativas e ao coeficiente de Spearman para a análise de associação entre variáveis ordinais. O valor de significância considerado foi de $5 \%$.

\section{RESULTADOS}

\section{Caracterização da Amostra}

Participaram 153 crianças e adolescentes, maioritariamente do sexo feminino (56,2\%), com média de idade de 9,9 $\pm 4,3$ anos. Verificou-se uma prevalência de excesso de peso e de obesidade de 21,6\% e $18,9 \%$, respetivamente, mais elevada no sexo feminino, ainda que sem significado estatístico ( $p=0,246)$. Do total dos inquiridos, $86,9 \%$ frequentavam a consulta de Pediatria e a maior parte dos questionários foi aplicado aquando de uma consulta subsequente $(77,8 \%$ ) (Tabela 1$)$.

\section{Adesão ao Padrão Alimentar Mediterrânico}

Através da análise do score final do questionário KIDMED foi possíve observar uma alta adesão ao PAM (81,0\% dos participantes) (Tabela 1). Na Tabela 2 podem observar-se as taxas de resposta às diferentes questões do índice KIDMED, sendo o consumo de frutos oleaginosos o item com menor pontuação $(22,9 \%)$.

\section{Tabela 1}

Caracterização da população $(n=153)$

\begin{tabular}{|c|c|c|c|}
\hline & & & $n(\%)$ \\
\hline \multirow{4}{*}{ Idade (anos) } & \multicolumn{2}{|l|}{$2-5$} & $26(17,0 \%)$ \\
\hline & \multicolumn{2}{|l|}{$5-10$} & $46(30,0 \%)$ \\
\hline & \multicolumn{2}{|l|}{$10-15$} & $57(37,0 \%)$ \\
\hline & \multicolumn{2}{|l|}{$15-18$} & $24(16,0 \%)$ \\
\hline \multirow{2}{*}{ Sexo } & \multicolumn{2}{|l|}{ Feminino } & $86(56,2 \%)$ \\
\hline & \multicolumn{2}{|l|}{ Masculino } & $67(43,8 \%)$ \\
\hline \multirow{4}{*}{$\begin{array}{l}\text { Tipo de } \\
\text { Consulta }\end{array}$} & \multirow{2}{*}{ Pediatria } & Primeira consulta & $31(23,3 \%)$ \\
\hline & & Subsequente & $102(76,7 \%)$ \\
\hline & \multirow{2}{*}{ Nutrição Pediátrica } & Primeira consulta & $3(15,0 \%)$ \\
\hline & & Subsequente & $17(85,0 \%)$ \\
\hline \multirow{5}{*}{$\begin{array}{l}\text { Estado } \\
\text { Nutricional }\end{array}$} & \multicolumn{2}{|l|}{ Desnutrição } & $3(2,0 \%)$ \\
\hline & \multicolumn{2}{|l|}{ Baixo Peso } & $11(7,2 \%)$ \\
\hline & \multicolumn{2}{|l|}{ Normoponderal } & $77(50,3 \%)$ \\
\hline & \multicolumn{2}{|l|}{ Excesso de Peso } & $33(21,6 \%)$ \\
\hline & \multicolumn{2}{|l|}{ Obesidade } & $29(18,9 \%)$ \\
\hline \multirow{3}{*}{$\begin{array}{l}\text { Adesão } \\
\text { ao Padrão } \\
\text { Alimentar } \\
\text { Mediterrânico }\end{array}$} & \multicolumn{2}{|l|}{ Alta Adesão } & $124(81,0 \%)$ \\
\hline & \multicolumn{2}{|l|}{ Adesão Intermédia } & $27(17,7 \%)$ \\
\hline & \multicolumn{2}{|l|}{ Baixa Adesão } & $2(1,3 \%)$ \\
\hline
\end{tabular}


Tabela 2

Tradução do Índice KIDMED e frequências absolutas e relativas das respostas às questões

\begin{tabular}{|c|c|c|}
\hline \multirow{2}{*}{ QUESTÕES } & SIM & NÃO \\
\hline & $\mathrm{n}(\%)$ & $\mathrm{n}(\%)$ \\
\hline 1. Comes uma peça de fruta ou bebes um sumo de fruta natural todos os dias? & $142(92,8 \%)$ & $11(7,2 \%)$ \\
\hline 2. Comes duas ou mais peças de fruta, todos os dias? & $110(71,9 \%)$ & $43(28,1 \%)$ \\
\hline 3. Comes vegetais crus (alface, tomate...) ou cozinhados (brócolos, couve...), regularmente, pelo menos uma vez por dia? & $137(89,5 \%)$ & $16(10,5 \%)$ \\
\hline 4. Comes vegetais crus (alface, tomate...) ou cozinhados (brócolos, couve...) mais do que uma vez por dia? & $96(62,7 \%)$ & $57(37,3 \%)$ \\
\hline 5. Comes peixe com regularidade (pelo menos 2 a 3 vezes por semana)? & $137(89,5 \%)$ & $16(10,5 \%)$ \\
\hline 6. Vais uma ou mais vezes por semana a restaurantes de fast-food? & $12(7,8 \%)$ & $141(92,2 \%)$ \\
\hline 7. Comes leguminosas (feijão, grão, ervilhas...) mais do que uma vez por semana? & $91(59,5 \%)$ & $62(40,5 \%)$ \\
\hline 8. Comes diariamente arroz ou massa ( 5 ou mais vezes por semana)? & $149(97,4 \%)$ & $4(2,6 \%)$ \\
\hline 9. Ao pequeno-almoço comes cereais ou derivados (pão...)? & $117(76,5 \%)$ & $36(23,5 \%)$ \\
\hline 10. Comes frutos oleaginosos (nozes, amêndoas...) pelo menos 2 a 3 vezes por semana? & $35(22,9 \%)$ & $118(77,1 \%)$ \\
\hline 11. Em tua casa, utilizam azeite? & $153(100 \%)$ & $0(0 \%)$ \\
\hline 12. Costumas avançar o pequeno-almoço? & $12(7,8 \%)$ & $141(92,2 \%)$ \\
\hline 13. Ao pequeno-almoço costumas beber leite ou derivados (iogurte, queijo...)? & $134(87,6 \%)$ & $19(12,4 \%)$ \\
\hline 14. Comes produtos de pastelaria ao pequeno-almoço? & $7(4,6 \%)$ & $146(95,4 \%)$ \\
\hline 15. Bebes diariamente 2 copos de leite/iogurtes ou 1 fatia grande de queijo? & $128(83,7 \%)$ & $25(16,3 \%)$ \\
\hline 16. Comes doces e guloseimas várias vezes ao dia? & $24(15,7 \%)$ & $129(84,3 \%)$ \\
\hline
\end{tabular}

A análise da adesão ao PAM em função do tipo de consulta frequentada, permitiu verificar uma alta adesão a este padrão pelas crianças e adolescentes que frequentavam quer a consulta de Pediatria quer a de Nutrição Pediátrica (80,5\% e 85,0\%, respetivamente; $p=0,314)$. Apesar da adesão ao PAM pelos participantes recrutados em contexto de primeira consulta ser inferior ao verificado por aqueles recrutados numa consulta subsequente, não se registaram diferenças com significado estatístico ( $p=0,103)$. O mesmo não se verificou quando se analisou a adesão a este modelo alimentar entre as crianças e adolescentes com excesso de peso/obesidade, registando-se uma maior adesão pelos participantes selecionados no âmbito de uma consulta subsequente (58,3\% versus $88,0 \% ; \mathrm{p}=0,008)$.

O nível de adesão ao PAM é similar entre sexos $(p=0,131)$. No entanto, registou-se uma forte associação com a idade, na medida em que o nível de adesão diminui com esta ( $r S p e a r m a n=0,279 ; p<0,001$ ). Embora pareça haver uma tendência para uma menor adesão ao PAM com o aumento do IMC, esta não revela ser significativa (rSpearman=0,106; $p=0,095)$. Também uma alta adesão a este padrão alimentar não parece estar relacionada com o estado nutricional das crianças e adolescentes (78,6\%, 80,5\% e 82,3\%, respetivamente para baixo peso/desnutrição, normoponderalidade e excesso de peso/obesidade; $p=0,469$ ).

Considerou-se pertinente analisar as diferentes questões da ferramenta KIDMED em função do estado nutricional dos participantes (dados não apresentados). Não se registaram diferenças com impacto em nenhuma das questões, com exceção da relativa à toma do pequeno-almoço (questão 12. Costumas avançar o pequeno-almoço?). Verificou-se que as crianças e adolescentes com excesso de peso ou obesidade eram as que, com mais frequência, omitiam esta refeição $(75,0 \%$; $p=0,014)$.

\section{DISCUSSÃO DOS RESULTADOS}

Ao longo dos últimos anos tem-se vindo a observar um progressivo afastamento do padrão alimentar tradicional, mais precisamente do PAM $(6,18)$. A globalização dos comportamentos e dos mercados, a mudança do paradigma da estrutura familiar e as modas poderão, pelo menos em parte, justificar esta mudança (19). A aplicação do índice KIDMED a um grupo de crianças e adolescentes que frequentam a consulta de Pediatria e de Nutrição Pediátrica de um hospital do norte de Portugal, permitiu verificar uma boa adesão a este padrão alimentar, resultados bem mais positivos do que os encontrados num estudo realizado em 2012, em crianças do sul do país (81.0\% versus $52,5 \%)(20)$.

É largamente reconhecido que uma elevada adesão à Dieta Mediterrânica se associa a um melhor estado nutricional, diminuição da prevalência de obesidade e de fatores de risco para a doença cardiovascular $(21,22)$. A população avaliada apresenta uma elevada prevalência de excesso de peso e obesidade (40,5\%), superior à registada para a população pediátrica portuguesa da mesma idade (cerca de 30\%) (9). A justificação poderá residir no facto das consultas onde se procedeu à aplicação do inquérito terem uma forte vertente de orientação nutricional e serem, em alguns casos, consulta de referência de obesidade pediátrica. No entanto, não se verificou qualquer relação entre a adesão ao PAM e o estado nutricional das crianças e adolescentes, tal como já observado por Arriscado e col. (23).

À semelhança de alguns estudos realizados em Itália e Espanha (23, 24), não foram encontradas diferenças na adesão ao PAM entre sexos. Já no que respeita à idade, verificou-se uma relação inversa à adesão ao PAM, isto é, quanto maior a idade, menor é a adesão a um padrão alimentar saudável e equilibrado. Este resultado não é totalmente inesperado se considerarmos que a adolescência constitui um período de maior autonomia nas escolhas alimentares comparativamente à infância, associado a mudança de comportamentos, invariavelmente com agravamento dos hábitos alimentares. Assim, a par da infância, a adolescência é igualmente um período prioritário de intervenção e de implementação de estratégias sustentáveis para melhoria do estilo de vida, através da adoção de práticas alimentares saudáveis e da prática de atividade física (24).

A adesão ao PAM entre as crianças e adolescentes que frequentavam os diferentes tipos de consulta (Pediatria e Nutrição Pediátrica) não diferiu significativamente. Constatou-se, sim, uma adesão bastante superior ao PAM nas consultas subsequentes entre aquelas que apresentavam excesso de peso ou obesidade, o que poderá traduzir um maior empenho na mudança de atitudes e comportamentos referentes à alimentação nesta população.

O consumo diário de um pequeno-almoço saudável e equilibrado é essencial no controlo da saciedade ao longo do dia e, consequentemente, na prevenção do ganho ponderal excessivo, o que sustenta os resultados 
obtidos, dado que as crianças e adolescentes com excesso de peso ou obesidade eram aquelas que, com mais frequência, omitiam esta refeição (25).

No contexto do PAM e do estilo de vida que the está associado, nomeadamente a prática de exercício físico, como limitações do presente trabalho poderá ser considerado o desconhecimento de dados relativos à prática de atividade física, os quais permitiriam correlacionar de forma mais precisa a adesão ao PAM e o estado nutricional das crianças e adolescentes. Outro aspeto, prende-se com o facto de ser um estudo realizado num hospital privado que, de certa forma, poderá traduzir-se numa amostra mais selecionada do ponto de vista socioeconómico e educacional. Para além disso, sendo um questionário de frequência alimentar, poderá resultar em sub ou sobrevalorização da informação relativa aos alimentos ingeridos.

\section{CONCLUSÕES}

Na população estudada, independentemente do sexo e do estado nutricional, a adesão ao PAM é elevada. No entanto, a adoção deste modelo alimentar decresce progressivamente com a idade, o que vem reforçar a necessidade da implementação precoce de estratégias eficazes de promoção de um estilo de vida saudável, ativo e tradicionalmente mediterrânico, incentivando o gosto e consumo de alimentos que caracterizam o PAM.

\section{REFERÊNCIAS BIBLIOGRÁFICAS}

1. Davis C, Bryan J, Hodgson J, Murphy K. Definition of the Mediterranean Diet: A Literature Review. Nutrients. 2015; 7(11): 9139-9153.

2. Graça P. Breve história do conceito de dieta Mediterrânica numa perspetiva de saúde. Fatores de Risco. 2014; 31: 20-22.

3. Barros V, Carrageta M, Graça P, Queiroz J, Sarmento M. Dieta Mediterrânica Um património civilizacional partilhado [Internet]. Comité Intergovernamental para a Salvaguarda do Património Cultural Imaterial da UNESCO; 2013 [cited 2019 Jul 1]. Available from: https://comum.rcaap.pt/handle/10400.26/10480.

4. Pinto A. Políticas para as doenças crónicas não transmissíveis em Portugal: uma retrospetiva desde a criação do serviço nacional de saúde. Contributos para uma política nutricional em Portugal. Tese Doutoramento Univ do Porto. 2016; 38 p.

5. Vilarnau C, Stracker DM, Funtikov A, Da Silva R, Estruch R, Bach-Faig A. Worldwide adherence to Mediterranean Diet between 1960 and 2011. Eur J Clin Nutr. 2019; 72, 83-91.

6. Pereira-da-Silva L, Rêgo C, Pietrobelli A. The Diet of Preschool Children in the Mediterranean Countries of the European Union: A Systematic Review. Int J Environ Res Public Health. 2016; 13(6).

7. Instituto Nacional de Saúde Doutor Ricardo Jorge (INSA). Childhood Obesity Surveillance Initiative: COSI Portugal 2019. Lisboa. 2019 [cited 2019 Sept 15]. Available from: http://www.insa.min-saude.pt/cosi-portugal-2019-excesso-de-pesoe-obesidade-infantil-continuam-em-tendencia-decrescente/.

8. Lopes C, et al. Inquérito Alimentar Nacional e de Atividade Física, IAN-AF 2015-2016: Relatório de resultados. Universidade do Porto, 2017. ISBN: 978-989-746-181-1. Available from: www.ian-af.up.pt.

9. DGS. Alimentação Saudável em Números. Programa Nacional para a Promoção da Alimentação Saudável. Lisboa: Direção-Geral da Saúde. 2014. [cited 2019 Jun 3]. Available from: https://www.dgs.pt/estatisticas-de-saude/estatisticas-de-saude/ publicacoes/portugal-alimentacao-saudavel-em-numeros-2014.aspx.

10. Castro-Quezada I, Román-Viñas B, Serra-Majem L. The Mediterranean Diet and Nutritional Adequacy: A Review. Nutrients. 2014; 6(1): 231-248.

11. Serra-Majem L, Ribas L, Ngo J, Ortega RM, García A, Pérez-Rodrigo C, Aranceta J. Food, youth and Mediterranean diet in Spain. Development of KIDMED, Mediterranean Diet Quality Index in children and adolescents. Public Health Nutr. 2004; 7(7): 931-935. 12. Koloverou E, Esposito K, Giugliano D, Panagiotakos D. The effect of Mediterranean diet on the development of type 2 diabetes mellitus: A meta-analysis of 10 prospective studies and 136,846 participants. Metabolism. 2014; 63(7): 903-911.

13. Kwan HY, Chao X, Su T, Fu X, Tse AK, Fong WF, Yu Z. The Anticancer and Antiobesity effects of Mediterranean diet. Crit Rev Food Sci Nutr. 2015; 57(1): 82-94. 14. Widmer RJ, Flammer AJ, Lerman LO, Lerman A. The Mediterranean Diet, its Components, and Cardiovascular Disease. Am J Med. 2015; 128(3): 229-238. 15. DGC, APN. Guia para educadores - Alimentação em idade escolar. Direção-Geral do Consumidor, Associação Portuguesa de Nutrição. 2013 [cited 2019 Jun 4]. Available from: https://www.apn.org.pt/documentos/guias/GuiaAPN_AlimentacaoldadeEscolar.pdf. 16. WHO Multicentre Growth Reference Study Group. WHO Child Growth Standards: Length/height-for-age, weight-for-age, weight-for-length, weight-for-height and body mass index-for-age: Methods and development. Geneva: WHO Library Cataloguingin-Publication Data; 2006 [cited 2019 Jun 18]. Available from: https://www.who.int/ childgrowth/standards/technical_report/en/.

17. Bach A, Serra-Majem L, Carrasco JL, Roman B, Ngo J, Bertomeu I, Obrador B. The use of indexes evaluating the adherence to the Mediterranean diet in epidemiological studies: a review. Public Health Nutr. 2006; 9(1a): 132-146.

18. Da Silva R, Bach-Faig A, Raidó Quintana B, Buckland G, Vaz de Almeida M, \& SerraMajem L. Worldwide variation of adherence to the Mediterranean diet, in 1961-1965 and 2000-2003. Public Health Nutrition. 2009; 12(9A), 1676-1684.

19. Pereira-da-Silva L, Pinto E. Baixa Adesão à Dieta Mediterrânica em Portugal: Nutrição das Grávidas em Portugal e suas Repercussões. Acta Med Port. 2016; 29(10):658-666.

20. Mateus M. Adesão ao Padrão Alimentar Mediterrânico em jovens no Algarve. Tese Doutoramento Univ do Porto. 2012; 84 p.

21. Galan-Lopez P, Ries F, Gisladottir T, Domínguez R, Sánchez-Oliver AJ. Healthy Lifestyle: Relationship between Mediterranean Diet, Body Composition and Physical Fitness in 13 to 16-Years Old Icelandic Students. Int J Environ Res Public Health. 2018; 15(12): 2632.

22. Yilmaz FÇ, Çağiran D, Özçelik AÖ. Adolescent Obesity and Its Association with Diet Quality and Cardiovascular Risk Factors. Ecol Food Nutr. 2019; 58(3): 207-218. 23. Arriscado D, Muros JJ, Zabala M, Dalmau JM. Factors associated with low adherence to a Mediterranean diet in healthy children in northern Spain. Appetite. 2014; 80: 28-34.

24. Grosso G, Marventano S, Buscemi S, Scuderi A, Matalone M, Platania A, Giorgianni G, Rametta S, Nolfo F, Galvano F, Mistretta A. Factors associated with adherence to the Mediterranean diet among adolescents living in Sicily, southern Italy. Nutrients. 2013; 5(12): 4908-4923.

25. Alsharairi NA, Somerset SM. Skipping breakfast in early childhood and its associations with maternal and child BMI: a study of 2-5-year-old Australian children. Eur J Clin Nutr. 2015; 70(4): 450-455. 